\title{
The Emerging Circadian Phenotype of Borderline Personality Disorder: Mechanisms, Opportunities and Future Directions
}

\author{
Niall M. McGowan ${ }^{1,2}$ (D) Kate E. A. Saunders ${ }^{1,2,3}$ (D) \\ Accepted: 23 February 2021 / Published online: 9 April 2021 \\ (C) The Author(s) 2021
}

\begin{abstract}
Purpose of Review We review the recent evidence suggesting that circadian rhythm disturbance is a common unaddressed feature of borderline personality disorder (BPD); amelioration of which may confer substantial clinical benefit. We assess chronobiological BPD studies from a mechanistic and translational perspective and highlight opportunities for the future development of this hypothesis.

Recent Findings The emerging circadian phenotype of BPD is characterised by a preponderance of comorbid circadian rhythm sleep-wake disorders, phase delayed and misaligned rest-activity patterns and attenuated amplitudes of usually well-characterised circadian rhythms. Such disturbances may exacerbate symptom severity, and specific maladaptive personality dimensions may produce a liability towards extremes in chronotype. Pilot studies suggest intervention may be beneficial, but development is limited. Summary Endogenous and exogenous circadian rhythm disturbances appear to be common in BPD. The interface between psychiatry and chronobiology has led previously to novel efficacious strategies for the treatment of psychiatric disorders. We believe that better characterisation of the circadian phenotype in BPD will lead to a directed biological target for treatment in a condition where there is a regrettable paucity of accessible therapies.
\end{abstract}

Keywords Circadian $\cdot$ Personality disorder $\cdot$ Actigraphy $\cdot$ Melatonin $\cdot$ Chronotype

\section{Introduction}

Borderline personality disorder (BPD) is a psychiatric disorder characterised by persistent mood instability, impulsivity, identity disturbance and interpersonal dysfunction. Among clinical populations BPD is the most commonly diagnosed personality disorder affecting $10 \%$ of psychiatric outpatients and between $15-25 \%$ of inpatients [1]. Functional impairment is substantial, involving turbulent interpersonal relationships, poor psychosocial and occupational outcomes [2, 3] and reckless and

This article is part of the Topical Collection on Sleep Disorders

Niall M. McGowan

niall.mcgowan@psych.ox.ac.uk

1 Department of Psychiatry, University of Oxford, Warneford Hospital, Oxford OX3 7JX, UK

2 Oxford Health NHS Foundation Trust, Warneford Hospital, Oxford, UK

3 NIHR Oxford Health Biomedical Research Centre, Oxford, UK potentially dangerous behaviours (e.g. substance abuse) [4]. Recurrent self-harm and suicidality are core symptoms, with up to $10 \%$ of individuals with BPD dying by suicide [4]. Treatments for BPD are limited. There are no medications specifically licenced for its treatment [5]. Long-term psychotherapies are efficacious [6], but not consistently available [7]. The biological phenotype of BPD has been largely neglected, hindering the innovation of novel translational interventions.

Sleep disturbance and insomnia are common in BPD [8••] but are under-appreciated compared to mood disorders where they form part of the diagnostic criteria. Consequently, the specific sleep complaints of people with BPD are often overlooked and thus untreated [9]. An important process governing sleep that remains under-investigated in BPD is that of the circadian clock. Circadian rhythms are 24-h physiological oscillations that sustain a temporal architecture of sleep/wake behaviour [10]. Circadian rhythm disturbances in behavioural and endocrine measures are implicated in the pathophysiology of several psychiatric disorders and are appreciated clinically as a driver of sleep disturbance [11-13]. Moreover, 
dimensional traits that also constitute core symptoms of BPD, such as mood instability and impulsivity, are associated with disturbance of circadian rest-activity patterns $[14,15]$. A role for altered circadian rhythm function has been proposed in BPD [16•], but to date, this hypothesis has not been elaborated further.

In this review, we apply a chronobiological perspective to the extant BPD sleep literature and review recent studies that specifically examine circadian rhythm function. First, we provide a brief primer on the circadian timing system for a general psychiatry audience. We examine the literature supporting the presence of physiologic circadian rhythm abnormalities in BPD, which consists of studies addressing comorbid circadian rhythm sleep-wake disorders, actigraphy monitoring, heartrate variability and endocrine measures and a small evidence base supporting chronotherapy for BPD. Given recent calls to articulate borderline pathophysiology dimensionally, we also discuss the association between chronotype and personality traits and the implication this raises for future BPD studies. Finally, we offer recommendations for future research development and clinical practice.

\section{The Circadian System}

\section{Circadian Entrainment and Circadian Rhythm Sleep Wake Disorders}

A schematic overview of circadian clock function is presented in Fig. 1 and a glossary of circadian terminology from this section (italicised terms in text) is presented in the 'Supplementary Information' section. The circadian system comprises a network of endogenously oscillating 24-h rhythms known as circadian rhythms that operate across multiple physiological and behavioural domains [18]. This system consists of multiple autonomous and self-sustaining oscillators throughout the brain and periphery. Hierarchical control over the circadian network is exerted by the central circadian pacemaker or 'master clock' in the suprachiasmatic nuclei (SCN) of the anterior hypothalamus [19]. The master clock aligns the phase of peripheral oscillators to a stable phase throughout the body, establishing a coordinated internal day of the circadian clock. Phase and amplitude are primary characteristics of the circadian rhythm. Phase measures the timing of the peak (or trough) of a circadian rhythm relative to a fixed event and is used to situate the rhythm within local time (or relative to sunrise, bedtime, etc.). Amplitude measures the difference between peak and trough, and thus quantifies the strength of the circadian output signal.

Entrainment is the process by which the circadian system synchronises its internal day relative to that of the environment [20]. Environmental agents that synchronise the circadian system are called zeitgebers. In absence of a zeitgeber signal, the internal day of the circadian clock oscillates with an intrinsic period that is close to, but deviates slightly from, 24-h; a condition known as free-running. Thus, in order to confer a functional advantage of anticipating environmental change, the circadian system establishes a stable phase relation or 'phase of entrainment' with the environment. Light is the predominant zeitgeber of the human circadian clock [21]. Several qualities of the light/dark cycle determine the expression of circadian rhythms such as the intensity, duration of exposure and time of day of exposure. The output circadian parameter signal may undergo modification of its amplitude (suppression/enhancement) and/or shift its phase (resulting in phase delay or phase advance).

According to the two-process model of sleep regulation, circadian rhythms interact with homeostatic factors to influence sleep onset time and consolidate a continuous sleep episode [10]. Sleep pressure (homeostatic process) accumulates during wake but is counterbalanced during the day by the circadian process, which exerts an opposing wake-promoting signal. Due to the circadian drive being rhythmic, this wake-promoting signal diminishes in the late evening, a time when homeostatic drive is also high, and in doing so facilitates sleep. Sleep promoting circadian signals involve rhythmic oscillations in core body temperature (CBT), autonomic nervous system (ANS) activity and endocrine rhythms (e.g. cortisol and melatonin) [22, 23], all of which are parameters that exhibit robust circadian rhythmicity even when free-running.

Recurrent abnormal phase relations between the circadian clock and the light/dark cycle produce a misalignment between sleep times and societal and physical 24-h schedules, extremes of which give rise to Circadian Rhythm Sleep Wake Disorders (CRSWDs) [24]. Advanced Sleep Wake Phase Disorder (ASWPD) and Delayed Sleep Wake Phase Disorder (DSWPD) refer to abnormally early and late timing of the sleep-wake interval relative to the socially desirable pattern, respectively. Individuals with DSWPD present with chronic sleep-onset insomnia and difficulty waking in the morning, resulting in excessive day-time sleepiness and educational/occupational impairment [25]. Conversely, individuals with ASWPD experience sleepiness in the late afternoon/early evening interfering with social activities, and may experience early-morning awakening insomnia or sleepmaintenance insomnia [25]. Non-24-h sleep-wake disorder (N24SWD) is a condition where a person is unable to entrain their circadian clock to the 24-h light/dark cycle; instead, the clock runs at its intrinsic period, which is typically longer than 24-h [26]. This results in a circadian pattern of sleep/wake scheduling that progressively and perpetually delays each day. N24SWD usually emerges through compromised retinohypothalamic signalling to the $\mathrm{SCN}$ and is common in blind people, but assumed very rare in sighted individuals $[26,27]$. 

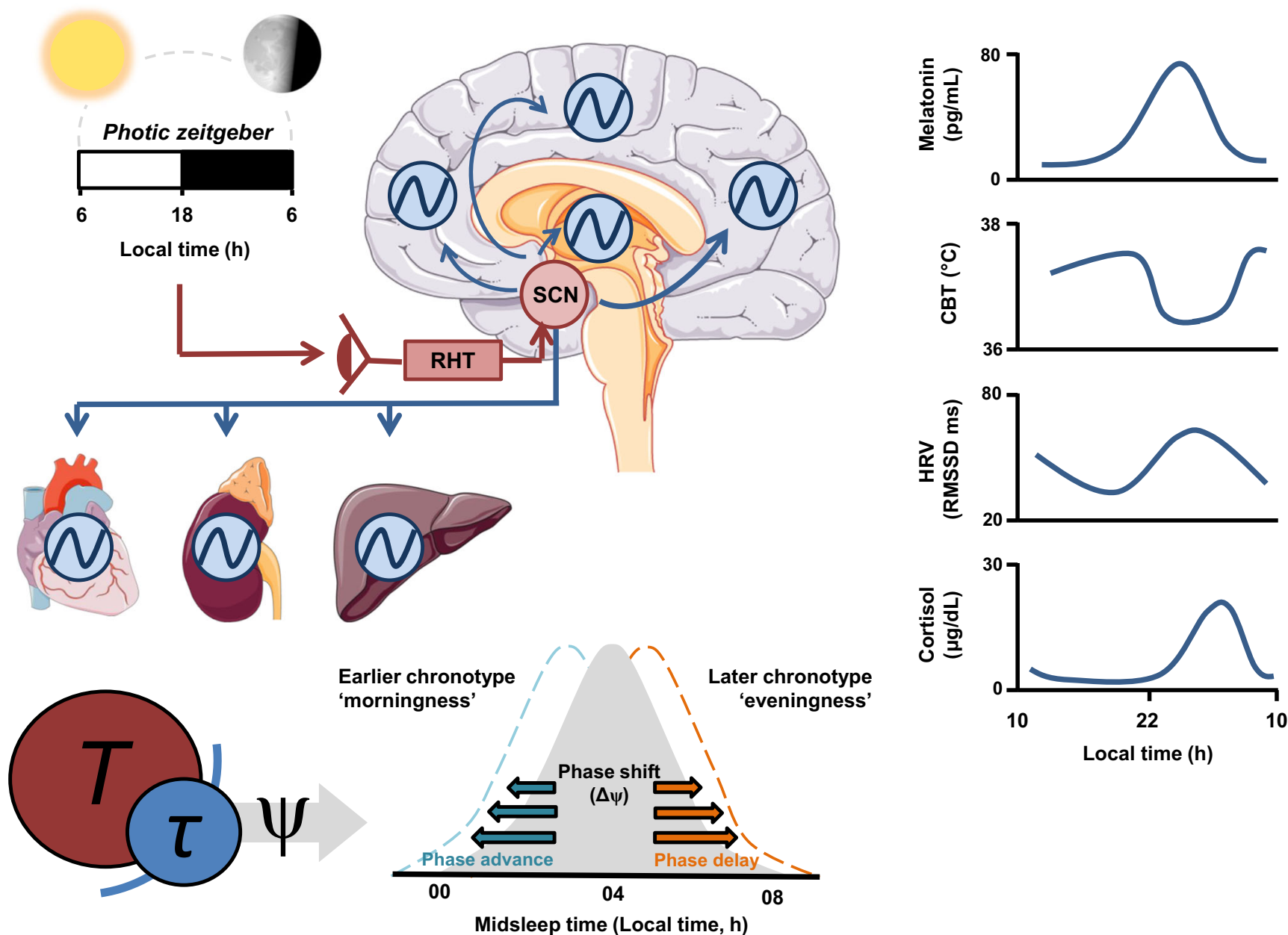

Fig. 1 Schematic of the circadian timekeeping system. The circadian time-keeping system is governed by the central clock of the SCN. Efferent SCN signals (blue) coordinate time-keeping among other circadian oscillators in the brain and peripheral organs, establishing a stable phase relation between endogenous circadian oscillators. This network generates an internally stable $24 \mathrm{~h}$ cycle that represents the internal day of the circadian system. Circadian signals used to determine the phase of the circadian clock involve robustly oscillating rhythms in core body temperature (CBT), inter-beat interval heart-rate variability (HRV) and plasma melatonin and cortisol. To impart a functional advantage of anticipating time related changes in the environment, the circadian clock is entrained to the external light-dark cycle which serves as the primary zeitgeber to the circadian clock. Retinal photic zeitgeber input signals (red) are transduced to the SCN via a dedicated non-visual circuit known as the retinohypothalamic tract (RHT). Hence, through entrainment, the SCN

\section{Assessment of Circadian Rhythms}

The activity of the circadian clock of the SCN cannot be observed directly in humans but its phase and amplitude can be assessed through strongly endogenous circadian rhythms such as are present in CBT and secretion of melatonin and cortisol [28]. Controlled laboratory studies involving constant routine or forced desynchrony protocols are used to observe circadian rhythms in absence of sleep and environmental masking, but are not common in psychiatric populations. Moreover, in situ

synchronises its period to that of the $24 \mathrm{~h}$ day. Interaction between the $24 \mathrm{~h}$ period of the external day (T) and that of the endogenous internal day (tau) generates the circadian phase of entrainment $(\psi)$. The phase range at which the internal day coalesces with the external $24 \mathrm{~h}$ day gives rise to different $\psi$ expressed as earlier or later chronotypes. Chronotype assessed via the midsleep time displays an approximately normal distribution in the population and is a trait that influences behavioural routines of rest and activity. Manipulation of zeitgeber exposure can produce phase shifts in $\psi$. Early morning exposure to bright light produces a phase advance of the circadian clock, whereas evening exposure produces a phase delay of the circadian clock. Using agents that adjust the phase of the circadian clock is the basis of chronotherapy. Endocrine and temperature reference ranges shown in figure are adapted from Hickie et al. [17] or based on laboratory estimates. RMSSD ranges are based on ambulatory ECG recordings

monitoring is desirable in order to capture circadian processes that pertain to the ecological experience of psychiatric disorder. Several non-invasive and ambulatory methods facilitate assessment in a normal day-to-day environment. Dim-light melatonin onset (DLMO) is the gold standard metric for determining the phase of the circadian clock and can be derived from saliva samples obtained from home at regular intervals under dim-light conditions [29]. Physiologic circadian rhythms can also be observed in cortisol secretion, and passive ambulatory methods such as distal/proximal temperature 
rhythms, and ANS activity assessed via 24-h ECG [28, 30, 31]. Furthermore, these measures can also be used to reflect the amplitude of the circadian rhythm, which melatonin profiles derived using the DLMO method do not capture.

Circadian rest-activity patterns can be monitored using actigraphy, which is a method widely used in psychiatry research where continuous monitoring of other circadian biomarkers may be prohibitive. A clear advantage of this method is assessment over multiple days, allowing longitudinal monitoring and parallel estimation of sleep [32]. The circadian phase of entrainment can be estimated using actigraph assessed acrophase, midsleep time or nonparametric circadian rhythm parameters [32, 33]. However, these methods are also susceptible to masking effects where activity that is used to infer properties of the underlying circadian rhythm is partially confounded by non-circadian phenomena (e.g. socially enforced schedules, abnormally high or low activity levels, device removal). Despite this caveat, actigraphy provides a useful objective estimate of circadian rhythm function where laboratory routines that examine endogenous clock parameters and eliminate masking effects are unavailable. Study design factors such as appropriate sampling and the use of simultaneous sleep dairies and device removal logs can improve the quality of actigraphy data.

Perhaps the most straightforward instantiation of the circadian phenotype is the emergence of circadian typologies or chronotypes, a trait reflecting either diurnal preference or selfselected sleep scheduling that is used to estimate the time of entrained circadian phase [20,34]. Chronotype is a dimensional trait ranging from morningness to eveningness, alternatively measured by early to late midsleep time. Chronotyping of individuals can be achieved quickly via questionnaires that enquire about daily habits and sleep. Instruments such as the Morningness-Eveningness Questionnaire (MEQ) [35] and Munich Chronotype Questionnaire (MCTQ) [36] are both self-reported methods for assessing chronotype that are validated against sleep diary data and objective measures of the circadian phase.

\section{Circadian Rhythm Disturbance in Borderline Personality Disorder}

Several lines of evidence from sleep studies suggest that circadian rhythm disturbance is a likely contributor to sleep disturbance in BPD. The most recent meta-analytic review of sleep findings in BPD identifies 32 studies [8••]. The most commonly employed method of objectively monitoring sleep in these studies was polysomnography (PSG) with 14 studies published since 1980. Overall, these reviewed findings indicate that sleep disturbance in BPD is characterised by longer sleep onset latency, reduced total sleep time and poor sleep efficiency. Self- reported sleep characteristics using questionnaire instruments also highlight sleep onset latency as a key problem [37-39]. Qualitative examination of BPD experiences reveals complaints of unstructured sleep patterns, and difficulty maintaining a stable sleep-wake routine with associated distress and occupational impairment [9]. The character of sleep disturbance is BPD is concordant with that of circadian phase delay (i.e. the nature of insomnia applies to sleep initiation rather than maintenance). Recently, Fitzpatrick et al. elaborated on these findings using the MEQ to assess chronotype [40॰]. In addition to describing greater insomnia severity in BPD compared to healthy controls, their study found greater trait eveningness in BPD compared to healthy controls and clinical controls (generalised anxiety disorder). Controlling for depressive symptoms and medication use suggested that chronotype differences in BPD may also depend on symptom severity.

People with BPD may have a higher risk of being diagnosed with a CRSWD. Dagan et al. reported in two studies a higher prevalence of DSWPD among individuals with personality disorder (including borderline) and vice versa [41, 42]. Early clinical reports describing sighted N24SWD cases have implicated borderline and schizoid psychopathology with the disorder [43, 44]. Reciprocally, individuals with DSWPD and N24SWD exhibit personality traits concordant with borderline personality features $[45,46]$. We are unaware of any reported association between ASWPD and BPD. Mechanistic understanding of how CRSWDs might relate to personality disorders is not yet elucidated. Poor entrainment to social zeitgebers, irregular work schedules or unemployment might contribute to DSWPD risk in BPD. For example, shift-work and unemployment may impact the clock by producing a recurring state of circadian misalignment and insufficient/mistimed zeitgeber exposure. Similarly, while the character and pathogenic mechanisms of N24SWD in sighted individuals remain poorly understood, social withdrawal and personality disorder are hypothesised pre-morbid factors [47, 48]. Accordingly, a disrupted sleep phenotype in BPD that is consistent with CRSWD may be overlooked by clinicians, considered secondary to personality disorder psychopathology and social/ functional impairment.

Cross-sectional studies directly monitoring circadian rhythms in BPD are limited in number. Verkes et al. were the first to describe abnormal rest-activity rhythms in a sample of majority BPD patients recruited from emergency department admissions [49]. Periodogram analysis of actigraphy data obtained over 5-7 consecutive days revealed a periodicity divergent from 24-h in almost two-thirds of participants. Non-24 $\mathrm{h}$ period was associated with worse borderline symptoms, suicidal ideation and disinhibition. Non-24 h activity periods are unusual outside of N24SWD, and in the case of normal day-to-day actigraphy assessment probably reflect between day variability of rest-activity timing rather than an abnormal rhythmic component per se [15]. Indeed, parallel 
sleep diary results found that improvement in bedtime regularity was associated with symptom improvement [49].

Huynh et al. examined actigraph patterns in an adolescent sample comprised of BPD and bipolar disorder (BD) [50॰]. Data were obtained over 9 consecutive days, containing two weekends to capture free-day versus school/work-day differences in behaviour. Overall, BPD participants exhibited more variable night-to-night sleep times compared to healthy controls. On free-days, BPD participants spent more time in bed and had a rise time over an hour later than controls. These findings suggest compensatory oversleeping on free-days consistent with social jetlag; a phenomenon that arises as a function of circadian rhythm misalignment with societal schedules [51]. However, given that the circadian clock is phase delayed during adolescence and social jetlag is more pronounced during school-age, caution is necessary when extrapolating these findings to adults. BPD is also not routinely diagnosed during adolescence.

One of the longest consecutive actigraphy assessment periods recorded in BPD was obtained during the Automated Monitoring of Symptom Severity (AMoSS) study [52]. $\mathrm{BPD}, \mathrm{BD}$ and healthy control groups were monitored using actigraphy for 28 days. BPD participants exhibited phase delayed rest patterns over 1:30 h later than BD and $\mathrm{HC}$, and phase delayed daytime activity patterns over $2 \mathrm{~h}$ later than HC [53••]. Cosinor analysis of distal skin temperature reflected similar delayed patterns in BPD. Furthermore, symptom severity in BPD was strongly associated with circadian rhythm disturbance [54••]. Impulsivity correlated with lower circadian rhythm amplitude and interdaily stability. Mood instability was associated with later activity onset, lower rhythm stability and amplitude, and greater rhythm fragmentation. Notably, mood instability in this study was derived from prospectively reported mood ratings collected for up to 12 months after initial actigraphy recording. Thus, unresolved circadian disturbance may be a predictor of enduring symptom severity. Due to the observational nature of these data, experimental studies are necessary to determine a causal relation between sleep/circadian disturbance and worsened symptom severity in BPD.

An earlier report on a sub-sample of AMoSS participants examined circadian patterns of heart-rate (HR) and activity over a 4 day assessment period using torso mounted holterlike devices containing accelerometer sensors [55•]. Cosinor acrophase of HR, total motor acceleration and vertical acceleration (estimating sleep) revealed desynchrony between HR and activity/sleep in BPD that was greater than BD and absent in controls. These findings suggest internal desynchrony between circadian oscillators in BPD, over and above BD. Moreover, in BPD, mood instability was associated with daily acrophase variability in HR, activity and sleep [56]. Limitations of this approach involve the sampling frequency; inter-beat intervals could not be assessed, and thus, standard time and frequency domain measures of heart-rate variability (HRV) could not be ascertained. Previous studies have examined HRV via ECG in BPD as a measure of ANS activity, but these have focused on short-term resting state recordings [57]. We are aware of only one study that examined 24-h ambulatory ECG in BPD [58]. However, to date, no studies have quantified the circadian rhythm of HRV in BPD.

A number of studies in BPD have examined endocrine parameters, which suggest circadian rhythm abnormality. Rausch et al. and Lieb et al. describe elevated salivary cortisol awakening response (CAR) in female BPD patients [59, 60]. The latter study also demonstrated diurnal elevation until the evening. Wingenfeld et al. report elevated overnight urinary free cortisol in BPD compared to controls [61]. JogemsKosterman et al. describe lower morning-to-evening salivary cortisol ratio in BPD compared to controls [62]. These findings suggest greater amplitude of cortisol secretion in response to awakening, and an attenuated 24-h rhythm in the context of elevated diurnal and nocturnal secretion. However, CAR and 24-h cortisol levels are not unambiguous measures of circadian phase or amplitude. The underlying circadian rhythm of cortisol is masked by an ultradian pulsatile secretion pattern that is likely activated further in individuals with alterations in hypothalamus-pituitary-adrenal axis function, as in BPD. The aforementioned cortisol pattern findings may be gender specific (female) and importantly have been shown in medication-free volunteers and controlling for comorbid depression and trauma [59•].

We are aware of only one study that examined melatonin circadian phase in BPD. Bromundt et al. examined melatonin secretion via consecutive saliva sampling during waking hours in a small sample of women (14 BPD compared with 10 healthy controls) [63・•]. Melatonin onset time and the phase angle between melatonin onset and bedtime did not differ between groups. Non-parametric indicators of circadian rest-activity function assessed through parallel 3week actigraph monitoring also showed no differences. As far as we know, no studies have used DLMO as a circadian phase marker in BPD.

Two studies have examined bright light therapy (BLT) in BPD, a treatment modality targeting circadian rhythm function. The aforementioned Bromundt et al. study used a 3-week cross-over design treatment of morning $8000 \mathrm{~lx}$ for 30-40 min daily [63••]. BLT led to earlier rise-time, shorter sleep duration and increased nocturnal proximal skin temperature (a circadian measure of vasodilation indexing preparedness for sleep). Daytime alertness and symptoms of atypical depression also improved with treatment. Prasko et al. examined 13 treatment resistant BPD patients with comorbid depression using BLT to augment antidepressant treatment [64•]. The treatment consisted of 6-week application of 1-h morning 10,000 lx BLT added to SSRI treatment. Symptoms of depression and anxiety improved with treatment. However, no 
measures of sleep or circadian function were employed. To date, there has been no randomised controlled trial of BLT or any other chronotherapeutic or sleep intervention in BPD.

\section{Dimensional Models of Borderline Personality, Symptoms and Chronotype}

The diagnosis of BPD is a categorical one made if five or more of nine behavioural criteria are present [65]. This variety of potential combinations contributes to variability in symptom aetiology, clinical course, and treatment response. That a categorical system may not be optimal for researching or diagnosing personality disorder is a long-standing issue in psychiatry [66]. Personality pathology can also be characterised dimensionally on a continuum, and the majority of the individual diagnostic criteria have been shown to be continuously distributed [67]. The move towards dimensional over categorical approaches to personality disorder classification is reflected in the hybrid dimensional-categorical model included for further study in DSM-5 and the dimensional approach taken in ICD-11. These developments have implications for how circadian rhythm differences may be expressed in BPD and their meaning with respect to symptom severity and management. Evidence from personality studies in the field of chronobiology suggest an association between circadian clock function and traits that predominate in BPD.

Normative personality traits reflected in the fivefactor model of personality (FFM) are among the most widely validated psychological phenotypes [68] and have been suggested as analogous dimensions in the study of maladaptive personality traits [69]. Metaanalytic studies of FFM traits in personality disorders highlight that BPD is characterised by high neuroticism, low agreeableness and low conscientiousness, resembling closely core symptoms of mood instability, interpersonal dysfunction and impulsivity, respectively [70, 71]. The overlap between these associations with delayed phase of entrainment suggested by chronotype studies is substantial. Two meta-analytic reviews conclude broadly similar findings. Later chronotype/ eveningness is associated with low conscientiousness and low agreeableness [72, 73•]. Furthermore, when morningness is considered a separate dimension on the MEQ (reflecting advanced phase of entrainment), a negative association with neuroticism emerges [73•].

Cloninger's Temperament and Character Inventory (TCI) [74] is an alternative personality model also commonly used in both BPD and chronobiology research. The TCI assesses four temperament dimensions (novelty seeking, harm avoidance, reward dependence and persistence) and three character dimensions (self-directedness, cooperativeness and self-transcendence). In BPD, temperament dimensions emphasise increased harm avoidance, increased novelty seeking and decreased reward dependence, and character dimensions emphasise decreased self-directedness and decreased cooperativeness, reflecting the clinical impression of the disorder [75, 76]. Chronotype studies indicate that eveningness is positively associated with novelty seeking with mixed findings for harm avoidance [77-81]. Furthermore, character findings suggest that eveningness is negatively associated with self-directedness, cooperation and persistence [77, 79-81].

In healthy populations, symptoms of BPD such as mood instability and impulsivity are both associated with circadian rest-activity pattern disturbance $[14,15]$. Eveningness/later chronotype is also a predictor of core BPD symptoms such as dissociative experiences [82], impulsivity [83], anger [84] and suicidal ideation [85]. Hypothesised mechanisms for these relations involve incompatibility between societal demands and chronotype (i.e. social jetlag) and the resultant detriment to sleep. However, there are no studies investigating chronotype and symptom dimensions in BPD and it is not clear how well findings from healthy participants can be extrapolated to BPD. Future chronotype and multimodal circadian phenotyping studies are needed in BPD with a focus on interrogating its polythetic personality and symptom domains.

\section{Future Research Opportunities and Clinical Recommendations}

The studies reviewed here indicate that circadian rhythm disturbance may be an important unmet treatment need for people with BPD and a factor that likely exacerbates the core symptoms of the disorder. It is our view that there is an unexploited opportunity to characterise and therapeutically address circadian rhythm disturbance in BPD, and that doing so may produce significant benefits for those with the diagnosis. We predict that interventions that stabilise circadian rhythm function in BPD will confer benefit for its treatment, particularly in those that exhibit delayed and disrupted sleep patterns. Studies examining the circadian phenotype in unipolar depression and bipolar disorder have demonstrated that circadian rhythm disturbances are a cardinal feature of the chronic sleep disturbance in both disorders $[17,86]$. Accordingly, chronotherapeutic interventions (such as BLT) and therapies designed to stabilise 24-h behavioural patterns (such as interpersonal and social rhythm therapy; IPSRT) have been deployed successfully for the treatment of both $[17,87,88]$. Circadian rhythm treatment represents a future directed biological target for intervention in BPD. In contrast to the prolonged nature of psychotherapeutic approaches, chronotherapeutic treatment response is achievable within weeks, and interventions are scalable and can be modified to be delivered remotely. Adjunctive to recommended psychotherapies, chronotherapeutic treatments may widen the treatment options that are available to people with BPD. 
Technological advances over the past decade have resulted in enthusiastic support for digital phenotyping in psychiatry; that is in situ monitoring and momentary quantification of individual-level phenotypes from smartphones and personal digital devices [89]. Behavioural circadian patterns are an additional target for future digital phenotyping studies and may be passively monitored using moment-to-moment digital device signals akin to actigraphy use in the field of chronobiology. Examining the impact of circadian rhythm disturbance and misaligned phase on symptom course, decompensation risk and impending crisis presents a novel opportunity for digital phenotyping studies in BPD.

There is also a yet unexplored opportunity to examine the association between dimensions of maladaptive personality and circadian phenotypes, notably chronotype, as has been richly described in the case of normative personality traits. Alternative DSM-5 Model for Personality Disorder [65] proposes a five factor dimensional maladaptive personality trait system that should be used in future studies. We suggest that future studies use a dimensional framework for understanding the impact of circadian rhythm disturbance upon BPD psychopathology. Based on the presentation of BPD and previous chronotype studies among clinical and general populations, it seems likely that domains such as negative affect and disinhibition are more severely impacted by circadian rhythm disturbance than symptoms involving complex interpersonal interactions. Delineation of susceptible features is necessary to innovate targeted approaches for symptom management.

We propose recommendations for future research of circadian clock function in BPD (Table 1) and highlight some clinical recommendations (Table 2). There is preliminary evidence of association between circadian rhythm disturbance and BPD diagnosis and psychopathology. However, this topic needs greater research attention and the causal direction of this relationship has yet to be determined. Future experimental and longitudinal work is necessary to elucidate the mechanisms

Table 1 Future research recommendations

- Obtain circadian parameters beyond actigraphy in BPD, focusing explicitly on physiologic markers of circadian phase and especially DLMO

- Design experimental medicine chronotherapy studies to examine causal hypotheses and provide clinical pilot data for future trials

- Determine the course of BPD symptomatology and how it relates to circadian rhythm disturbance via longitudinal ecological momentary assessment studies

- Delineate symptom features and dimensional pathological personality traits that are adversely affected by circadian rhythm disturbance in BPD

- Sleep studies should at a minimum employ parallel measures of chronotype to capture information about the phase of sleep/wake patterns in BPD
Table 2 Clinical recommendations

- Sleep should be routinely assessed in personality disorders and detailed psychiatric interview should be conducted to screen for comorbid CRSWDs

- Consider sleep disturbance and circadian rhythm misalignment as perpetuating and exacerbating factors of core borderline symptoms, especially in the context of occupational and social impairment

- Emphasise sleep hygiene and social rhythm stabilisation as strategies supplementary to treatment as usual

involved and to track the evolution of symptom severity. An experimental medicine approach will be useful for both establishing the impact of circadian clock function on core BPD symptoms and assessing the efficacy of chronotherapeutic interventions in the disorder. Appreciation for a role of circadian rhythm disturbance in BPD may enable translational therapies for its treatment as has been demonstrated for bipolar disorder [88], a condition with considerable symptom overlap. Feasibility studies in BPD have demonstrated that BLT may be a promising therapeutic avenue $[63,64]$ that should be further interrogated in a randomised controlled manner.

Fundamental questions concerning the character of circadian function in BPD remain unaddressed. Most studies have used actigraphy to assess rest-activity rhythms but assessment of other circadian parameters is required. Endocrine and ANS activity measures are variables of interest in BPD studies and express a circadian rhythm that should be examined explicitly. Chronobiological studies have established the DLMO phase of the circadian clock in other psychiatric disorders but this has not yet been examined in BPD. As the purported gold standard marker of clock function, its characterisation will allow important questions to be clarified involving internal phase angle coordination and internal day representation that may be interrupted in BPD.

Clinical practice points should involve routine assessment of sleep disorder in BPD with a particular focus on CRSWDs given their preponderance and reciprocal nature. Sleep complaints from people with BPD should not be considered secondary to core psychopathology. Treatment of sleep disturbance and abnormal sleep/wake phase should be explicit and above usual symptom management for BPD. Until specific evidence-based chronotherapeutic interventions for BPD become available, sleep hygiene and IPSRT inspired approaches with psychoeducation regarding appropriate zeitgeber exposure and social rhythm regularity should be emphasised.

\section{Conclusions}

There is a growing evidence base suggesting circadian rhythm dysregulation in BPD. Recent findings indicate that rest-activity patterns are phase delayed and possibly 
desynchronised internally from other oscillating autonomic parameters. Reduced circadian rhythm amplitude and daily stability of rest-activity and endocrine rhythms have also recently been reported and these perturbations appear to be associated with worsened core symptoms of the disorder. Chronotype studies in normative samples show consistent associations between later chronotype and traits associated with borderline psychopathology but this trend requires further examination in those with a confirmed diagnosis of BPD. Taken together, the emerging circadian phenotype of BPD suggests a phase delayed clock comporting with earlier clinical reports that describe a greater prevalence of a delayed sleep-wake phase syndrome among people with the disorder. However, further development of this hypothesis is necessary using unambiguous endogenous measures of the clock function (e.g. DLMO). Better classification of the circadian rhythm phenotype in BPD will permit a full characterisation of the impact on patient impairment and enable the development of targeted interventions. Pilot studies find some initial support for BLT but the feasibility of other chronotherapies is not yet clear.

\section{Footnote}

\section{Search Strategy and Selection Criteria}

We selected references for this article from previous work in the field of chronobiology and via a search of AMED, Embase, MEDLINE/Pubmed, PsycINFO and Google Scholar published up until May 31, 2020, using the search terms 'borderline personality disorder' or 'emotionally unstable personality disorder' and 'circadian', 'chronotype', 'biological clock', 'actigraphy', 'actimetry', 'cortisol' and 'melatonin'. From these searches, we identified articles published in English and reviewed the citations within these articles. We determined a final list of references based on their relevance to the theme of this review.

Supplementary Information The online version contains supplementary material available at https://doi.org/10.1007/s11920-021-01236-w.

Acknowledgements We attribute credit for the brain, heart, kidney and liver artwork adapted for use in Fig. 1 to SMART Servier Medical Art (https://smart.servier.com/). These images are publically available and supplied freely for redistribution and adaptation under a Creative Commons Attribution 3.0 Unported Licence. These images have been adapted by the authors of this article and their use does not reflect an endorsement of the content of this article by the licensor.

Author Contribution NMMcG and KEAS developed the initial concept of the article. $\mathrm{NMMcG}$ conducted the literature search, interpreted and critically appraised relevant study findings, made the figures and tables and wrote the first draft of manuscript. KEAS critically revised the first draft. Both authors approved the final draft.
Funding Dr. Saunders is supported by the NIHR Oxford Health Biomedical Research Centre.

\section{Declarations}

Conflict of Interest The authors declare that they have no conflict of interest.

Human and Animal Rights This article does not contain any studies with human or animal subjects performed by any of the authors.

Disclaimer The views expressed are those of the authors and not necessarily those of the NHS, the NIHR or the Department of Health.

Open Access This article is licensed under a Creative Commons Attribution 4.0 International License, which permits use, sharing, adaptation, distribution and reproduction in any medium or format, as long as you give appropriate credit to the original author(s) and the source, provide a link to the Creative Commons licence, and indicate if changes were made. The images or other third party material in this article are included in the article's Creative Commons licence, unless indicated otherwise in a credit line to the material. If material is not included in the article's Creative Commons licence and your intended use is not permitted by statutory regulation or exceeds the permitted use, you will need to obtain permission directly from the copyright holder. To view a copy of this licence, visit http://creativecommons.org/licenses/by/4.0/.

\section{References}

Papers of particular interest, published recently, have been highlighted as:

- Of importance

•. Of major importance

1. Ellison WD, Rosenstein LK, Morgan TA, Zimmerman M. Community and clinical epidemiology of borderline personality disorder. Psychiatr Clin North Am. 2018;41(4):561-73.

2. Zanarini MC, Frankenburg FR, Reich DB, Fitzmaurice G. The 10year course of psychosocial functioning among patients with borderline personality disorder and axis II comparison subjects. Acta Psychiatr Scand. 2010 Aug;122(2):103-9.

3. Hengartner MP, Müller M, Rodgers S, Rössler W, Ajdacic-Gross V. Occupational functioning and work impairment in association with personality disorder trait-scores. Soc Psychiatry Psychiatr Epidemiol. 2014;49:327-35.

4. Leichsenring F, Leibing E, Kruse J, New AS, Leweke F. Borderline personality disorder. Lancet. 2011;377(9759):74-84.

5. Hancock-Johnson E, Griffiths C, Picchioni M. A focused systematic review of pharmacological treatment for borderline personality disorder. CNS Drugs. 2017;31(5):345-56.

6. Cristea IA, Gentili C, Cotet CD, Palomba D, Barbui C, Cuijpers P. Efficacy of psychotherapies for borderline personality disorder: a systematic review and meta-analysis. JAMA Psychiatry. 2017;74(4):319-28.

7. Dale O, Sethi F, Stanton C, Evans S, Barnicot K, Sedgwick R, et al. Personality disorder services in England: findings from a national survey. BJ Psych Bull. 2017;41(5):247-53.

8.• Winsper C, Tang NK, Marwaha S, Lereya ST, Gibbs M, Thompson A, et al. The sleep phenotype of Borderline Personality Disorder: a systematic review and meta-analysis. 
Neurosci Biobehav Rev. 1(73):48-67. Most recent and comprehensive systematic review of sleep studies in BPD.

9. Wood A, Brooks R, Beynon-Pindar C. The experience of sleep for women with borderline personality disorder: an occupational perspective. Br J Occup Ther. 2015 Dec;78(12):750-6.

10. Borbély AA, Daan S, Wirz-Justice A, Deboer T. The two-process model of sleep regulation: a reappraisal. J Sleep Res. 2016 Apr;25(2):131-43.

11. Alloy LB, Ng TH, Titone MK, Boland EM. Circadian rhythm dysregulation in bipolar spectrum disorders. Curr Psychiatry Rep. 2017 Apr;19(4):21.

12. Vadnie CA, McClung CA. Circadian rhythm disturbances in mood disorders: insights into the role of the suprachiasmatic nucleus. Neural plasticity. 2017;2017:1-28.

13. Coogan AN, McGowan NM. A systematic review of circadian function, chronotype and chronotherapy in attention deficit hyperactivity disorder. Atten Defic Hyperact Disord. 2017;9(3):129-47.

14. Rock P, Goodwin G, Harmer C, Wulff K. Daily rest-activity patterns in the bipolar phenotype: a controlled actigraphy study. Chronobiol Int. 2014;31(2):290-6.

15. McGowan NM, Coogan AN. Sleep and circadian rhythm function and trait impulsivity: an actigraphy study. Psychiatry Res. 2018;268:251-6.

16. Fleischer M, Schäfer M, Coogan A, Häßler F, Thome J. Sleep disturbances and circadian CLOCK genes in borderline personality disorder. J Neural Transm. 2012;119(10):1105-10. First review of empirical circadian studies relevant to BPD. This review suggests putative involvement of circadian dysfunction.

17. Hickie IB, Naismith SL, Robillard R, Scott EM, Hermens DF. Manipulating the sleep-wake cycle and circadian rhythms to improve clinical management of major depression. BMC medicine. 2013;11(1):1-27.

18. Pilorz V, Helfrich-Förster C, Oster $\mathrm{H}$. The role of the circadian clock system in physiology. Pflugers Arch. 2018 Feb 1;470(2): 227-39.

19. Patton AP, Hastings MH. The suprachiasmatic nucleus. Curr Biol. 2018 Aug 6;28(15):R816-22

20. Roenneberg T, Merrow M. The circadian clock and human health. Curr Biol. 2016 May 23;26(10):R432-43.

21. Dijk DJ, Archer SN. Light, sleep, and circadian rhythms: together again. PLoS Biol. 2009;7(6):e1000145.

22. Saper CB, Scammell TE, Lu J. Hypothalamic regulation of sleep and circadian rhythms. Nature. 2005;437(7063):1257-63.

23. Stehle JH, Von Gall C, Korf HW. Melatonin: a clock-output, a clock-input. J Neuroendocrinol. 2003;15(4):383-9.

24. Sateia MJ. International classification of sleep disorders. Chest. 2014;146(5):1387-94.

25. Reid KJ, Zee PC. Circadian rhythm sleep disorders. Handb Clin Neurol. 2011;99:963-77.

26. Malkani RG, Abbott SM, Reid KJ, Zee PC. Diagnostic and treatment challenges of sighted non-24-hour sleep-wake disorder. J Clin Sleep Med. 2018;14(04):603-13.

27. Sack RL, Lewy AJ, Blood ML, Keith LD, Nakagawa HI. Circadian rhythm abnormalities in totally blind people: incidence and clinical significance. J Clin Endocrinol Metab. 1992;75(1):127-34.

28. Hofstra WA, de Weerd AW. How to assess circadian rhythm in humans: a review of literature. Epilepsy Behav. 2008;13(3):43844.

29. Burgess HJ, Wyatt JK, Park M, Fogg LF. Home circadian phase assessments with measures of compliance yield accurate dim light melatonin onsets. Sleep. 2015;38(6):889-97.

30. Krauchi K, Deboer T. The interrelationship between sleep regulation and thermoregulation. Front Biosci. 2010;15:604-25.

31. Boudreau P, Yeh WH, Dumont GA, Boivin DB. Circadian variation of heart rate variability across sleep stages. Sleep. 2013;36(12): 1919-28.
32. Ancoli-Israel S, Cole R, Alessi C, Chambers M, Moorcroft W, Pollak CP. The role of actigraphy in the study of sleep and circadian rhythms. Sleep. 2003;26(3):342-92.

33. Van Someren EJ, Swaab DF, Colenda CC, Cohen W, McCall WV, Rosenquist PB. Bright light therapy: improved sensitivity to its effects on rest-activity rhythms in Alzheimer patients by application of nonparametric methods. Chronobiol Int. 1999;16(4):505-18.

34. Adan A, Archer SN, Hidalgo MP, Di Milia L, Natale V, Randler C. Circadian typology: a comprehensive review. Chronobiol Int. 2012;29(9):1153-75.

35. Horne JA, Östberg O. A self-assessment questionnaire to determine morningness-eveningness in human circadian rhythms. Int J Chronobiol. 1976;4:97-110.

36. Roenneberg T, Wirz-Justice A, Merrow M. Life between clocks: daily temporal patterns of human chronotypes. J Biol Rhythm. 2003;18(1):80-90.

37. Plante DT, Frankenburg FR, Fitzmaurice GM, Zanarini MC. Relationship between sleep disturbance and recovery in patients with borderline personality disorder. J Psychosom Res. 2013;74(4):278-82.

38. Selby EA. Chronic sleep disturbances and borderline personality disorder symptoms. J Consult Clin Psychol. 2013;81(5):941-7.

39. Taherifard M, Abolghasemi A, Hajloo N. Positive and negative urgency and sleep quality among patients with borderline and antisocial personality disorders. Arch Psychiatry Psychother. 2015;17(4):105-12.

40. Fitzpatrick S, Maich KHG, Carney CE, Kuo JR. Identifying specific insomnia components in borderline personality disorder and their influence on emotion dysregulation. Personal Disord. 2020. https:// doi.org/10.1037/per0000395. This sleep study additionally examines chronotype in BPD compared to healthy and clinical control groups.

41. Dagan Y, Sela H, Omer H, Hallis D, Dar R. High prevalence of personality disorders among circadian rhythm sleep disorders (CRSD) patients. J Psychosom Res. 1996;41(4):357-63.

42. Dagan Y, Stein D, Steinbock M, Yovel I, Hallis D. Frequency of delayed sleep phase syndrome among hospitalized adolescent psychiatric patients. J Psychosom Res. 1998;45(1):15-20.

43. Wollman M, Lavie P. Hypernychthemeral sleep-wake cycle: some hidden regularities. Sleep. 1986;9(2):324-34.

44. Kokkoris CP, Weitzman ED, Pollak CP, Spielman AJ, Czeisler CA, Bradlow $\mathrm{H}$. Long-term ambulatory temperature monitoring in a subject with a hypernychthemeral sleep-wake cycle disturbance. Sleep. 1978;1(2):177-90.

45. Wilhelmsen-Langeland A, Saxvig IW, Pallesen S, Nordhus IH, Vedaa $\varnothing$, Sørensen E, et al. The personality profile of young adults with delayed sleep phase disorder. Behav Sleep Med. 2014;12(6): 481-92.

46. Micic G, Lovato N, Gradisar M, Lack LC. Personality differences in patients with delayed sleep-wake phase disorder and non-24-h sleep-wake rhythm disorder relative to healthy sleepers. Sleep Med. 2017;30:128-35.

47. Hayakawa T, Uchiyama M, Kamei Y, Shibui K, Tagaya H, Asada $\mathrm{T}$, et al. Clinical analyses of sighted patients with non-24-hour sleep-wake syndrome: a study of 57 consecutively diagnosed cases. Sleep. 2005;28(8):945-52.

48. Okawa M, Uchiyama M. Circadian rhythm sleep disorders: characteristics and entrainment pathology in delayed sleep phase and non24 sleep-wake syndrome. Sleep Med Rev. 2007;11(6):485-96.

49. Verkes RJ, Kerkhof GA, Beld E, Hengeveld MW, van Kernpen GM. Suicidality, circadian activity rhythms and platelet serotonergic measures in patients with recurrent succidal behaviour. Acta Psychiatr Scand. 1996;93(1):27-34.

50. Huỳnh C, Guilé JM, Breton JJ, Godbout R. Sleep-wake patterns of adolescents with borderline personality disorder and bipolar disorder. Child Psychiatry Hum Dev. 2016;47(2):202-14. This 
actigraphy study is the first suggesting greater social jetlag in adolescents with BPD.

51. Wittmann M, Dinich J, Merrow M, Roenneberg T. Social jetlag: misalignment of biological and social time. Chronobiol Int. 2006;23(1-2):497-509.

52. Tsanas A, Saunders KE, Bilderbeck AC, Palmius N, Osipov M, Clifford GD, et al. Daily longitudinal self-monitoring of mood variability in bipolar disorder and borderline personality disorder. J Affect Disord. 2016;205:225-33.

53.• McGowan NM, Goodwin GM, Bilderbeck AC, Saunders KE. Circadian rest-activity patterns in bipolar disorder and borderline personality disorder. Transl Psychiatry. 2019;9(1):1-1. Cross-sectional actigraphy and temperature monitoring period in BPD indicate that rest-activity patterns are phase-delayed in BPD compared to healthy controls and volunteers with bipolar disorder.

54.• McGowan NM, Goodwin GM, Bilderbeck AC, Saunders KE. Actigraphic patterns, impulsivity and mood instability in bipolar disorder, borderline personality disorder, and healthy controls. Acta Psychiatr Scand. 2020;141(4):374-84. This report indicates strong associations between weak/unstable and delayed circadian rest-activity patterns and longitudinally monitored symptoms of impulsivity and mood instability in BPD.

55. Carr O, Saunders KE, Bilderbeck AC, Tsanas A, Palmius N, Geddes JR, et al. Desynchronization of diurnal rhythms in bipolar disorder and borderline personality disorder. Transl Psychiatry. 2018;8(1):1-9. Parallel heart-rate and activity monitoring suggest internal desynchrony of physiologic circadian rhythms in BPD.

56. Carr O, Saunders KE, Tsanas A, Bilderbeck AC, Palmius N, Geddes JR, et al. Variability in phase and amplitude of diurnal rhythms is related to variation of mood in bipolar and borderline personality disorder. Sci Rep. 2018;8(1):1-1.

57. Koenig J, Kemp AH, Feeling NR, Thayer JF, Kaess M. Resting state vagal tone in borderline personality disorder: a meta-analysis. Prog Neuro-Psychopharmacol Biol Psychiatry. 2016;64:18-26.

58. Ebner-Priemer UW, Welch SS, Grossman P, Reisch T, Linehan MM, Bohus M. Psychophysiological ambulatory assessment of affective dysregulation in borderline personality disorder. Psychiatry Res. 2007;150(3):265-75.

59. Rausch J, Gäbel A, Nagy K, Kleindienst N, Herpertz SC, Bertsch K. Increased testosterone levels and cortisol awakening responses in patients with borderline personality disorder: gender and trait aggressiveness matter. Psychoneuroendocrinology. 2015;55:116-27. The largest and most recent study demonstrating elevated cortisol awakening response in medication-free BPD participants with age and gender matched controls.

60. Lieb K, Rexhausen JE, Kahl KG, Schweiger U, Philipsen A, Hellhammer DH, et al. Increased diurnal salivary cortisol in women with borderline personality disorder. J Psychiatr Res. 2004;38(6): 559-65.

61. Wingenfeld K, Driessen M, Adam B, Hill A. Overnight urinary cortisol release in women with borderline personality disorder depends on comorbid PTSD and depressive psychopathology. Eur Psychiatry. 2007;22(5):309-12.

62. Jogems-Kosterman BJ, De Knijff DW, Kusters R, van Hoof JJ. Basal cortisol and DHEA levels in women with borderline personality disorder. J Psychiatr Res. 2007;41(12):1019-26.

63.• Bromundt V, Wirz-Justice A, Kyburz S, Opwis K, Dammann G, Cajochen C. Circadian sleep-wake cycles, well-being, and light therapy in borderline personality disorder. J Personal Disord. 2013;27(5):680-96. First study to examine melatonin secretion in BPD. Pilot study also suggests clinical benefits derived from bright light therapy in BPD.

64. Prasko J, Brunovsky M, Latalova K, Grambal A, Raszka M, Vyskocilova J, et al. Augmentation of antidepressants with bright light therapy in patients with comorbid depression and borderline personality disorder. Biomed Pap Med Fac Univ Palacky Olomouc Czech Repub. 2010;154(4):355-61. First reported application of bright light therapy in BPD. Reports improvements in depression and anxiety symptoms.

65. American Psychiatric Association. Diagnostic and statistical manual of mental disorders. 5th ed. (DSM-5), (American Psychiatric Publishing, Arlington; 2013).

66. Frances A. Categorical and dimensional systems of personality diagnosis: a comparison. Compr Psychiatry. 1982;23:516-27.

67. Clark LA, Livesley WJ, Morey L. Personality disorder assessment: the challenge of construct validity. J Personal Disord. 1997;11(3): 205-31.

68. McCrae RR, John OP. An introduction to the five-factor model and its applications. J Pers. 1992;60(2):175-215.

69. Trull TJ, Widiger TA. Dimensional models of personality: the fivefactor model and the DSM-5. Dialogues Clin Neurosci. 2013;15(2): $135-46$.

70. Saulsman LM, Page AC. The five-factor model and personality disorder empirical literature: a meta-analytic review. Clin Psychol Rev. 2004;23(8):1055-85.

71. Samuel DB, Widiger TA. A meta-analytic review of the relationships between the five-factor model and DSM-IV-TR personality disorders: a facet level analysis. Clin Psychol Rev. 2008;28(8): $1326-42$.

72. Tsaousis I. Circadian preferences and personality traits: a metaanalysis. Eur J Pers. 2010;24(4):356-73.

73. Lipnevich AA, Credè M, Hahn E, Spinath FM, Roberts RD, Preckel F. How distinctive are morningness and eveningness from the Big Five factors of personality? A meta-analytic investigation. J Pers Soc Psychol. 2017;112(3):491. Comprehensive systematic review and meta-analysis on the association of chronotype with FFM personality dimensions.

74. Cloninger CR, Przybeck TR, Svrakic DM, Wetzel RD. The temperament and character inventory (TCI): a guide to its development and use. St. Louis: Washington University Center for Psychobiology of Personality; 1994.

75. Cloninger CR. Psychobiology and treatment of borderline personality disorder. Acta Neuropsychiatr. 2002;14(2):60-5.

76. Barnow S, Herpertz SC, Spitzer C, Stopsack M, Preuss UW, Grabe HJ, et al. Temperament and character in patients with borderline personality disorder taking gender and comorbidity into account. Psychopathology. 2007;40(6):369-78.

77. Adan A, Lachica J, Caci H, Natale V. Circadian typology and temperament and character personality dimensions. Chronobiol Int. 2010;27(1):181-93.

78. Caci H, Robert P, Boyer P. Novelty seekers and impulsive subjects are low in morningness. Eur Psychiatry. 2004;19(2):79-84.

79. Randler C, Saliger L. Relationship between morningnesseveningness and temperament and character dimensions in adolescents. Personal Individ Differ. 2011;50(2):148-52.

80. Antúnez JM, Navarro JF, Adan A. Morningness-eveningness and personality characteristics of young healthy adults. Personal Individ Differ. 2014;68:136-42.

81. Lee K, Lee HK, Jhung K, Park JY. Relationship between chronotype and temperament/character among university students. Psychiatry Res. 2017;251:63-8.

82. Selvi Y, Kandeger A, Boysan M, Akbaba N, Sayin AA, Tekinarslan E, et al. The effects of individual biological rhythm differences on sleep quality, daytime sleepiness, and dissociative experiences. Psychiatry Res. 2017;256:243-8.

83. McGowan NM, Voinescu BI, Coogan AN. Sleep quality, chronotype and social jetlag differentially associate with symptoms of attention deficit hyperactivity disorder in adults. Chronobiol Int. 2016;33(10):1433-43. 
84. Jankowski KS, Linke M. Angry night birds: emotionality, activity and sociability temperament in adolescent chronotypes. Chronobiol Int. 2020;23:1-8.

85. Selvi Y, Aydin A, Boysan M, Atli A, Agargun MY, Besiroglu L. Associations between chronotype, sleep quality, suicidality, and depressive symptoms in patients with major depression and healthy controls. Chronobiol Int. 2010;27(9-10):1813-28.

86. Melo MC, Abreu RL, Neto VB, de Bruin PF, de Bruin VM. Chronotype and circadian rhythm in bipolar disorder: a systematic review. Sleep Med Rev. 2017;34:46-58.

87. Al-Karawi D, Jubair L. Bright light therapy for nonseasonal depression: meta-analysis of clinical trials. J Affect Disord. 2016;198:64 71.
88. Gottlieb JF, Benedetti F, Geoffroy PA, Henriksen TE, Lam RW, Murray G, et al. The chronotherapeutic treatment of bipolar disorders: a systematic review and practice recommendations from the ISBD task force on chronotherapy and chronobiology. Bipolar Disord. 2019;21(8):741-73.

89. Torous J, Kiang MV, Lorme J, Onnela JP. New tools for new research in psychiatry: a scalable and customizable platform to empower data driven smartphone research. JMIR Ment Health. 2016;3(2):e16.

Publisher's Note Springer Nature remains neutral with regard to jurisdictional claims in published maps and institutional affiliations. 